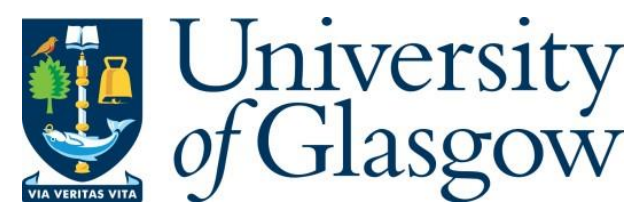

Kansal, A. R. et al. (2017) Financial impact of ivabradine on reducing heart failure penalties under the Hospital Readmission Reduction Program. Current Medical Research and Opinion, 33(2), pp. 185-191.

There may be differences between this version and the published version. You are advised to consult the publisher's version if you wish to cite from it.

http://eprints.gla.ac.uk/137464/

Deposited on: 1 March 2017

Enlighten - Research publications by members of the University of Glasgow http://eprints.gla.ac.uk 


\section{Financial Impact of Ivabradine on Reducing Heart Failure Penalties Under the Hospital Readmission Reduction Program}

Anuraag R. Kansala, Stanimira Krotneva ${ }^{a}$, Ali Tafazzolia, Harshali K. Patel ${ }^{\mathrm{b}}$, Jeffrey S. Borer $^{c}$, Michael Böhm ${ }^{d}$, Michel Komajda ${ }^{e}$, Juan Maya ${ }^{b}$, Luigi Tavazzi ${ }^{\dagger}$, Ian Ford ${ }^{\mathrm{g}}$, Adrian Kielhorn ${ }^{b}$

aEvidera, Bethesda, MD, USA; ${ }^{b}$ Amgen Inc., Thousand Oaks, CA, USA; 'Division of Cardiovascular Medicine, The Howard Gilman Institute for Heart Valve Diseases and Ronald and Joan Schiavone Cardiovascular Translational Research Institute, State University of New York Downstate Medical Center, Brooklyn and New York, NY, USA; dKlinik für Innere Medizin III, Universitätsklinikum des Saarlandes, 66424

Homburg/Saar, Germany; eDepartment of Cardiology, Pitié-Salpétrière Hospital, University Pierre et Marie Curie and IHU ICAN, Paris, France; ' Maria Cecilia Hospital, GVM Care \& Research, Ettore Sansavini Health Science Foundation, Cotignola, Italy; gRobertson Centre for Biostatistics, University of Glasgow, Glasgow, Scotland

Article type: original article

Correspondence to Harshali Patel, Manager of Cardiovascular Global Health

Economics, One Amgen Center Drive, 28-3-A, Thousand Oaks, CA 91320, USA.

Phone: 805-447-1947. E-mail: harshali@amgen.com

Short title: Ivabradine reduces heart failure readmission penalties

Previous presentations: these data have not been presented elsewhere but will be submitted as an abstract to TBD 


\section{ABSTRACT}

Objective: The introduction of the Hospital Readmission Reduction Program (HRRP) has led to renewed interest in developing strategies to reduce 30-day readmissions among patients with heart failure (HF). In this study, a model was developed to investigate whether the addition of ivabradine to a standard-of-care (SoC) treatment regimen for patients with HF would reduce HRRP penalties incurred by a hypothetical hospital with excess 30-day readmissions.

Research design and methods: A model using a Monte Carlo simulation framework was developed. Model inputs included national hospital characteristics, hospital-specific characteristics, and the ivabradine treatment effect as quantified by a post hoc analysis of the Systolic Heart failure treatment with the $\underline{I}_{f}$ inhibitor ivabradine Irial (SHIFT). Results: The model computed an $83 \%$ reduction in $\mathrm{HF}$ readmission penalty payments in a hypothetical hospital with a readmission rate of $22.95 \%$ (excess readmission ratio $=$ 1.056 over the national average readmission rate of $21.73 \%$ ), translating into net savings of $\$ 44,016$. A sensitivity analysis indicated that the readmission penalty is affected by the specific characteristics of the hospital, including the readmission rate, size of the ivabradine-eligible population, and ivabradine utilization. Conclusions: The results of this study indicate that the addition of ivabradine to an SoC treatment regimen for patients with HF may lead to a reduction in the penalties incurred by hospitals under the HRRP. This highlights the role ivabradine can play as part of a wider effort to optimize the care of patients with HF.

Keywords: ivabradine; readmissions; heart failure; hospital readmission reduction program (HRRP); readmission penalties 


\section{Introduction}

The Hospital Readmission Reduction Program (HRRP) was added to the Affordable

Care Act in 2012 to provide a direct financial incentive for hospitals participating in the Inpatient Prospective Payment System (IPPS) to reduce readmission rates ${ }^{1,2}$. The program implements penalties based on 30-day all-cause readmissions for five conditions: heart failure (HF), acute myocardial infarction, pneumonia, chronic obstructive pulmonary disease, and hip or knee replacement. An excess readmission is expressed as a ratio, and is defined for each hospital by dividing a hospital's number of predicted 30-day readmissions by the number that would be expected based on an average hospital with similar patients: a ratio $>1$ would indicate excess readmissions for a hospital. The HRRP operates on a 3-year rolling average basis, with penalties administered with a 1-year delay. For example, an HRRP penalty for a hospital with excessive readmissions in 2016 would reflect the hospital's performance over the 3-year period from July 2011 to July 2014 . A hospital with higher than national average readmissions can be penalized annually at a maximum of $3 \%$ of its Medicare diagnosisrelated group (DRG) payments; therefore, reducing 30-day readmission rates has become an important objective for US hospitals looking to avoid financial penalties.

To this end, many hospitals have implemented general strategies to reduce 30-day readmissions, with an emphasis on improving care both during and after discharge ${ }^{3}$. Predischarge interventions include improvements in patient education, discharge planning, and medication reconciliation, and scheduling follow-up appointments before discharge. Interventions designed to aid in the transition from hospital to home include 
providing patient-centered discharge instructions, using transition coaches, and attempting to ensure same-provider continuity between inpatient and outpatient care ${ }^{3}$. Postdischarge strategies include timely communication with primary care physicians, appropriate clinic follow-up, and follow-up telephone calls with patients ${ }^{3}$. It has also been well documented that the use of such a multitude of strategies is required to have a significant impact on readmission rates ${ }^{4,5}$.

Of the five conditions included in the HRRP, HF is the leading cause of 30-day readmissions: $22 \%$ of patients are readmitted within this timeframe ${ }^{6}$. The need for readmission has remained high despite the significant improvements made within the last two decades in the treatment of HF, including the introduction of therapies such as beta-blockers, angiotensin receptor blockers, angiotensin-converting enzyme (ACE) inhibitors, and aldosterone antagonists ${ }^{7}$. Consequently, there is significant interest in developing additional strategies to minimize readmissions following HF-associated hospitalization to not only control costs, but to also improve patients' quality of life. In fact, quality of life was assessed in a pre-specified substudy of SHIFT and demonstrated significant improvement in quality of life during a among patients treated with ivabradine vs those treated with placebo when assessed at 4 months, 12 months and last post-baseline visit ${ }^{8}$. This analysis did not specifically assess the impact of reduction in hospitalizations, per se, on quality of life; such an assessment is appropriate for future research. However, the majority of this research has been conducted on the use of multidisciplinary strategies spanning education, postdischarge care and monitoring, and communication between hospitals and community care 
providers, with little emphasis on how specific medical interventions or medication use can improve readmission rates ${ }^{9-11}$.

In patients with $\mathrm{HF}$, a relatively high resting heart rate $(\mathrm{HR})(\geq 70 \mathrm{bpm})$ has been identified as a modifiable risk factor that is associated with an increased risk of hospitalization and rehospitalization ${ }^{12-14}$. Ivabradine (Corlanor; Amgen Inc., Thousand Oaks, CA), a specific inhibitor of the If current in the sinoatrial node that reduces HR without affecting ventricular repolarization or myocardial contractility, was approved in April 2015 by the US Food and Drug Administration to reduce the risk of hospitalization for worsening HF in patients with stable, symptomatic, chronic HF with left-ventricular ejection fraction $\leq 35 \%$, who are in sinus rhythm with a resting $\mathrm{HR} \geq 70 \mathrm{bpm}$ and who either are receiving maximally tolerated doses of or have a contraindication to betablockers ${ }^{15}$. This approval was based on the results of the $\underline{\text { Systolic }}$ Heart failure treatment with the $\underline{\mathbf{I}}_{\mathbf{f}}$ inhibitor ivabradine $\underline{\text { Trial }}$ (SHIFT), which showed that treatment with ivabradine in combination with guideline-based standard-of-care (SoC) therapy was associated with a relative risk reduction of $11 \%$ for all-cause hospitalization and $26 \%$ for HF-related hospitalization ${ }^{16}$. A subsequent post hoc analysis of the SHIFT data also indicated that treatment with ivabradine led to a substantial reduction in the risk of rehospitalization for worsening $\mathrm{HF}^{17}$; therefore, ivabradine represents a potential strategy for reducing 30-day readmission rates for patients with HF. Because the effect of ivabradine on 30-day readmissions and the resulting potential reductions in financial penalties associated with these readmissions has not yet been investigated, the aim of this study was to develop a model for a hypothetical hospital with excess readmissions 
to evaluate the potential change in HRRP penalties associated with adding ivabradine to SoC therapy, compared with SoC alone.

\section{Methods}

\section{Overview}

A Monte Carlo simulation model was developed to quantify the potential financial impact of ivabradine on reducing all-cause 30-day readmissions following an HF admission for a hypothetical hospital under the HRRP. The model first computed the total HRRP penalties for the hospital, based on its rates of excess readmission. The model then applied the utilization rate of ivabradine and the effect of ivabradine on 30-day readmission rates to calculate the reduction in readmission associated with the addition of ivabradine to SoC therapy. Finally, the model translated the reduction in 30-day readmission with ivabradine into a reduction in the HRRP penalty.

\section{Model structure}

The HRRP model simulated the rate of 30-day readmission with and without ivabradine for a hypothetical hospital in the middle of the penalty range. Figure 1 shows the distribution readmission rates of the hospitals participating in the HRRP, as published by the Centers for Medicare \& Medicaid Services (CMS): the hospitals above the national average $(21.73 \%)$ are penalized. For the purpose of this study, the example hospital in the middle of the penalty range, which eventually is at the $75^{\text {th }}$ percentile. To account for the distribution of the potential effects of ivabradine on readmission rates, the model used a Monte Carlo simulation framework (1000 replications) that estimated the range of potential reductions in excess readmissions and HRRP penalties. Each replication of the Monte Carlo simulation took a sample from the readmission rate 
reduction distribution, and then adjusted the readmission rate reduction for ivabradine compared with SoC to compute an effective reduced hospital readmission rate. If the reduction did not bring the 30-day readmission rate below the national average (the point at which the penalty is completely eliminated), the model computed the reduced HRRP penalty with the adjusted readmission rate based on the HRRP algorithm published by the $\mathrm{CMS}^{1,2}$.

\section{Model inputs}

The model inputs were divided into three groups: national-level characteristics, hospitalspecific characteristics (for the example hospital), and treatment effect of ivabradine. The model simulated HRRP penalties for a hypothetical hospital with and without the use of ivabradine. Thus, the model input the characteristics of the hypothetical hospital, compared these characteristics to the national average, and then applied the treatment effect of ivabradine on readmissions. The hypothetical hospital was assumed to reflect the national average in terms of case mixture and reimbursements, with a readmission rate at the middle $\left(75^{\text {th }}\right.$ percentile) among hospitals penalized for readmission following HF admission.

\section{National hospital characteristics}

The national total annual DRG payments for all discharges for 2010-2014 were taken from the Medicare Payment Advisory Commission data on Medicare total payment for fee-for-service beneficiaries for inpatient services in acute care hospitals ${ }^{1,2}$; these data were used to compute the national average annual DRG payment for all discharges, which was $\$ 117$ billion. The national payment per HF discharge was computed from data provided in the CMS acute IPPS final rule and the national average payment table 
from OPTUM/INGENIX for $2010-2013^{18}$, and was used to estimate the national average payment per HF discharge, which was $\$ 6285$. The national total number of HF discharges was 1,097,144 and the national average readmission rate was $21.73 \%$; these were estimated based on the latest HRRP data provided by CMS from July 2011 to July 2014 (Table 1).

\section{Hospital-specific characteristics}

Readmission penalties are dependent on the hospital's excess readmission ratio. The excess readmission ratio was determined by the specific characteristics of the hospital being evaluated. For the hypothetical hospital in this analysis, the excess readmission ratio was selected using an all-cause readmission rate of $22.95 \%$, which was divided by the national average HF readmission rate of $21.73 \%$. Thus, the excess readmission ratio for the hypothetical study hospital was 1.056 , which was around the 75 th percentile of national distribution. The HRRP model estimated the hospital's total annual payments for all discharges by computing its ratio relative to the national average total annual DRG payments. Furthermore, the hypothetical hospital was assumed to have 150 annual HF admissions, and its total annual HF DRG payment was estimated by multiplying its annual HF admission with the national average payment per HF discharge (\$6285). For the purpose of this model, an ivabradine utilization scenario of $100 \%$ among readmitted patients was assumed. The size of the eligible patient population and ivabradine utilization rate were chosen to simplify interpretation of the base-case results (Table 1) and then tested in sensitivity analyses. In summary, the hypothetical hospital with an excess readmission ratio of 1.056 closely corresponded to 
the median hospital in the penalty range for HF. The HRRP hospital readmission rates provided by the CMS for 2011-2014 are shown in Figure 1.

\section{Treatment effect of ivabradine}

To estimate the impact of ivabradine on 30-day all-cause readmission rate reduction, a post hoc analysis of SHIFT data was conducted. A Bayesian approach was chosen to quantify the probability of a reduction in readmission over a range of effect sizes and to study the effect of uncertainty on reduction in HRRP penalties. This approach was selected because it focuses directly on the probability of observing a given treatment effect based on the available data, rather than on disproving a hypothesis of no treatment effect. The model then uses the full range of potential treatment effects weighted by their computed probabilities instead of a single point estimate, providing more flexibility in describing the range of potential benefit or cost. Bayes theorem assigns a probability to a hypothesis directly and incorporates prior available information (as probabilities), which is then updated with the new evidence. For the purpose of this analysis, noninformative prior distributions were used; in other words, the results of the analysis were informed by SHIFT data only. All Institutional Review Boards at the each of the participating sites approved the study and all patients provided consent. All inferences were made based on the posterior distributions; the mean provided a point estimate, and the $2.5^{\text {th }}$ and $97.5^{\text {th }}$ percentiles were used to create $95 \%$ credible intervals (Crls). All analyses were performed using the GENMOD procedure with the Bayes statement in SAS version 9.4 (SAS Institute Inc., Cary, NC, USA). 
The post hoc analyses using patient-level SHIFT trial data for 30-day all-cause readmission yielded a rate difference $(\mathrm{RD})(95 \% \mathrm{Crl})$ of $-1.97 \%(-6.29,2.39)$ for ivabradine compared with placebo, ie, the use of ivabradine was associated with avoiding 30 -day readmissions in a mean of $1.97 \%$ of patients with an initial HF-related hospitalization. The probability of observing an $\mathrm{RD}$ that was negative (ie, a reduction of some magnitude in 30 -day readmissions) was $82 \%$, while the probability of observing an RD of less than $-2 \%$ was $50 \%$ (Table 2). The cumulative distribution function of allcause readmission rate reduction for ivabradine compared with SoC treatment is shown in Figure 2.

\section{Model assumptions}

As with any model, the HRRP model relied on a set of assumptions. Most importantly, the analysis also assumed that the absolute reduction in 30-day all-cause readmission rate with ivabradine estimated using SHIFT data would translate to the real-world US clinical practice setting. In the absence of US-specific data for ivabradine's effect on readmissions, this assumption was selected to be more conservative (ie, less favorable) to ivabradine. Because 30-day readmission rates in the US claims data are higher compared with those in SHIFT ${ }^{1,2,17}$, a relative risk-based approach would have resulted in a larger absolute treatment effect. The potential effect of ivabradine was only considered on readmissions for patients who had an initial hospitalization: its effect on initial HF admissions was not included. This was also a conservative assumption regarding the effect of ivabradine on penalties, as a reduction in the number of patients at risk of readmission would also lower the absolute readmission penalty. 
Finally, it was assumed that the ivabradine utilization and rate of all-cause readmissions would be constant over the 3-year time horizon from which readmission rates are averaged in the HRRP. These assumptions were made to simplify the analyses and presentation of results, and do not alter the results for any individual year. Similarly, the model assumed that the penalties for the HRRP conditions other than HF would remain unaffected by ivabradine over this period. Unless a hospital is at or near the ceiling for the overall HRRP penalty, changes in the non-HF components would not affect the HF penalty.

\section{Results}

\section{HRRP penalty reduction results}

After applying the reduction in 30-day readmissions (absolute $-2 \%$ reduction with a probability of $50 \%$; corresponding to preventing three readmissions in the example hospital with 150 initial HF admissions) due to ivabradine, the excess readmission ratio for the example hospital was reduced from 1.056 to 1.0093 (Table 3). This reduction in excess readmission ratio translates into an $83 \%$ reduction in HF readmission penalty payments in the example hospital. The $83 \%$ reduction in penalty equates to $\$ 44,016$, which would be incurred over 3 years when applying the median readmission rate reduction with ivabradine (Table 4).

\section{Sensitivity analyses}

The fraction by which the penalty would be reduced decreases for a hospital with a higher rate of excess readmissions, as the effects of ivabradine are considered to be an absolute RD (Figure 3). If a hospital has an excess readmission ratio of 1.2 , the model predicts an HRRP penalty reduction of $25 \%$. As the excess readmission rate gets closer 
to 1 , the proportion of penalty reduction approaches $100 \%$. Figure 4 illustrates a tornado diagram on reduction in HRRP penalty for three scenarios: ivabradine utilization (50\%, $100 \%)$, ivabradine eligibility ( $25 \%, 75 \%)$, and cohort selection in the post hoc SHIFT analyses (excluding no patients, except for patients who died during the index hospitalization). In the base case, 100\% (upper bound) ivabradine utilization was considered where the penalty reduction was $\sim 83 \%$. When the ivabradine utilization is reduced to $50 \%$ (lower bound), in the one-way sensitivity analyses the penalty reduction would be $42 \%$. In other words, hospitals with smaller ivabradine-eligible populations or with lower utilization of ivabradine would similarly have smaller predicted reductions in the penalty.

\section{Discussion}

The model developed within this analysis indicates that the addition of the HR-lowering drug ivabradine to an SoC treatment regimen for patients with HF could substantially reduce the penalty incurred by a hospital under the HRRP, though this benefit may be specific to patients with HF, given that ivabradine did not affect hospitalization for newonset or worsening of HF in patient populations defined as chronic stable coronary artery disease not meeting the inclusion criteria for SHIFT ${ }^{19}$. It should be noted that the sensitivity analysis indicated that the readmission penalty was affected by the specific characteristics of each hospital, and therefore that the estimated effect on the penalty only directly applies to the hypothetical hospital considered here; yet, the results likely indicate a potential real-world benefit. To our knowledge, this is the first study that has been carried out to analyze the potential financial benefits of adding ivabradine to an SoC regimen for patients with HF in light of the penalties implemented under the HRRP. 
The introduction of the HRRP has intensified interest into strategies that can be used to reduce 30-day readmissions, particularly for patients with HF due to their high readmission rate. A number of general strategies have been shown to have some success in reducing readmission rates, including partnering with community physicians, partnering with local hospitals, having nurses perform medication reconciliation, arranging follow-up appointments before discharge, having a process in place to send all relevant discharge information to the patient's primary care physician, and assigning staff to follow-up on test results that return following discharge ${ }^{10}$. However, due to the variable implementation of such strategies and the large number of factors involved in readmission, the benefits of introducing such interventions has remained modest ${ }^{5,9}$. In contrast to these general interventions, the model developed within this study considered a strategy highly specific to patients with HF. The results indicate that addition of ivabradine to an $\mathrm{SoC}$ regimen has the potential to reduce penalties incurred under the HRRP, and suggest that HF-specific medication-based strategies may also improve readmission rates. While several other studies investigating the benefits of medications have suggested that diuretics, mineralocorticoid antagonists, ACE inhibitors, and digoxin may also improve readmission rates, none have modeled the potential financial benefits of their use in light of the HRRP $20-22$.

While the model developed herein suggests that the addition of ivabradine to an SoC regimen may avoid penalties by reducing readmission rates, it must be emphasized that the implementation of this strategy in conjunction with other, more general strategies will 
likely provide more substantial cost savings. This can be assumed on the basis that the hospitals that are most successful in reducing readmission rates have implemented multiple strategies, with the greatest improvements seen with those using three or more ${ }^{4,23}$. Future studies should consider the addition of ivabradine to an SoC regimen as part of a system-wide approach that utilizes a range of risk-reduction strategies. Whether the inclusion of a medication-based strategy could provide benefits beyond those achieved using general strategies alone, is also worth considering.

This model has a number of strengths. Firstly, an appropriate statistical approach was used to extract the probability distribution for an ivabradine effect on readmission rates from the SHIFT data. As mentioned earlier, a Bayesian approach was selected because it focuses directly on the probability of observing a given treatment effect based on the available data, rather than on demonstrating that the effect is likely to be nonzero. Therefore, the model uses the full range of potential treatment effects weighted by their computed probabilities, rather than a single point estimate. Utilizing the full probability distribution for changes in 30-day readmission rate weighted by a probability of its occurrence allows for a more complete picture of the range of potential outcomes an individual hospital might expect. The second strength of the model is that the readmission penalty was computed in the model following the CMS procedure for an individual hospital and using US hospital readmission data, which have the most direct relevance to the hospital. 
However, there are several limitations. The 30-day readmission practices used in this analysis may vary from that the real-world setting, as may the clinical characteristics of the patients within the SHIFT study; yet ivabradine has been available to a wider patient population in Europe since 2005, and the tolerability profile remains remarkably unchanged ${ }^{24}$. The model also relates to the assumption of $100 \%$ utilization of ivabradine in readmitted patients-in the real world, it would be unlikely for all patients who would be readmitted within 30 days of the initial HF hospitalization discharge to be eligible for ivabradine treatment. There is a lack of literature regarding specific demographic and clinical characteristics of patients who are readmitted, and therefore a $100 \%$ utilization rate was chosen to simplify interpretation of the base-case results, and then, a range was tested in sensitivity analyses. Finally, it should also be noted that the readmission penalty is applied on a 3-year rolling basis, and changes in the utilization of ivabradine or the eligible population over the 3-year rolling period would alter its impact on the penalty. A real-world analysis would validate these findings. Changes in the national landscape may also influence the penalty assessed, even if there are no changes at an individual hospital.

\section{Conclusion}

The results of this study indicate that reducing 30-day readmissions by adding ivabradine to an SoC treatment regimen may lead to a reduction in the penalties incurred by hospitals under the HRRP. This highlights the role ivabradine can play as part of a wider effort to optimize the care of patients with HF. 


\section{Transparency}

\section{Declaration of funding}

Funding for the SHIFT study was provided by Servier Inc., and funding for this analysis was provided by Amgen Inc.

\section{Declaration of financial/other relationships}

JSB has disclosed scientific support, consulting fees, and/or speaker honoraria from Servier and Amgen Inc., and consulting fees from Celladon, Pfizer, ARMGO, Cardiorentis, Novartis, AstraZeneca, and Takeda USA. MB, IF, and MK have disclosed scientific support, consulting fees, and/or speaker honoraria from Servier and Amgen Inc. ARK, SK, and AT are employees of Evidera. HKP, AK, and JM are employees of and stockholders in Amgen Inc.

\section{Acknowledgments}

Editorial support was provided by Janice Carlson, PhD, of Amgen Inc. and Shobana Ganesan, MSc, CACTUS Communications, on behalf of Amgen Inc. 


\section{References}

1. Readmissions reduction program (HRRP). Baltimore, MD: Centers for Medicare \& Medicaid Services. Available at: https://www.cms.gov/Medicare/Medicare-Fee-forService-Payment/AcutelnpatientPPS/Readmissions-Reduction-Program.html [2 February 2016]

2. Inpatient PPS PC pricer. Baltimore, MD: Centers for Medicare \& Medicaid Services. Available at: https://www.cms.gov/medicare/medicare-fee-for-servicepayment/pcpricer/inpatient.html [2 February 2016]

3. Hansen LO, Young RS, Hinami K, et al. Interventions to reduce 30-day rehospitalization: a systematic review. Ann Intern Med 2011;155:520-8

4. Kripalani S, Theobald CN, Anctil B, Vasilevskis EE. Reducing hospital readmission rates: current strategies and future directions. Annu Rev Med $2014 ; 65: 471-85$

5. Bradley EH, Curry L, Horwitz LI, et al. Contemporary evidence about hospital strategies for reducing 30-day readmissions: a national study. J Am Coll Cardiol 2012;60:607-14

6. Readmissions and Deaths - National. Data.Medicare.gov. Available at: https://data.medicare.gov/Hospital-Compare/Readmissions-and-Deaths-National/qqw3t4ie\# [1 February 2016]

7. Gheorghiade M, Vaduganathan M, Fonarow GC, Bonow RO. Rehospitalization for heart failure: problems and perspectives. J Am Coll Cardiol 2013;61:391-403

8. Ekman I, Chassany $\mathrm{O}$, Komajda $\mathrm{M}$, et al. Heart rate reduction with ivabradine and health related quality of life in patients with chronic heart failure: results from the SHIFT study. European Heart J 2011;32:2395-404 
9. Dharmarajan K, Hsieh AF, Lin Z, et al. Hospital readmission performance and patterns of readmission: retrospective cohort study of Medicare admissions. BMJ 2013;347:f6571

10. Bradley EH, Curry L, Horwitz LI, et al. Hospital strategies associated with 30-day readmission rates for patients with heart failure. Circ Cardiovasc Qual Outcomes 2013;6:444-50

11. Feltner $\mathrm{C}$, Jones $\mathrm{CD}$, Cené $\mathrm{CW}$, et al. Transitional care interventions to prevent readmissions for persons with heart failure: a systematic review and metaanalysis. Ann Intern Med 2014;160:774-84

12. DeVore AD, Schulte PJ, Mentz RJ, et al. Relation of elevated heart rate in patients with heart failure with reduced ejection fraction to one-year outcomes and costs. Am J Cardiol 2016;117:946-51

13. Diaz A, Bourassa MG, Guertin M-C, Tardif J-C. Long-term prognostic value of resting heart rate in patients with suspected or proven coronary artery disease. Eur Heart J 2005;26:967-74

14. Habal MV, Liu PP, Austin PC, et al. Association of heart rate at hospital discharge with mortality and hospitalizations in patients with heart failure. Circ Heart Fail 2014;7:12-20

15. Corlanor [package insert]. Thousand Oaks, CA: Amgen Inc.; 2015

16. Swedberg K, Komajda M, Böhm M, et al; on behalf of the SHIFT Investigators. Ivabradine and outcomes in chronic heart failure (SHIFT): a randomised placebocontrolled study. Lancet 2010;376:875-85 
17. Borer JS, Böhm M, Ford I, et al; on behalf of the SHIFT Investigators. Effect of ivabradine on recurrent hospitalization for worsening heart failure in patients with chronic systolic heart failure: the SHIFT Study. Eur Heart J 2012;33:2813-20

18. INGENIX, a comprehensive guidebook to the MS-DRG classification system, appendix E. National Average Payment Table. DRG Expert 2010-2013. Available at: https://www.optum360coding.com/upload/pdf/6097/DRG11.pdf. [Last accessed 12 March 2015]

19. Fox K, Ford I, Steg PG, et al. Ivabradine for patients with stable coronary artery disease and left-ventricular systolic dysfunction (BEAUTIFUL): a randomised, double-blind, placebo-controlled trial. Lancet 2008;372:807-16.

20. Flather MD, Yusuf S, Køber L, et al; for the ACE-Inhibitor Myocardial Infarction Collaborative Group. Long-term ACE-inhibitor therapy in patients with heart failure or left-ventricular dysfunction: a systematic overview of data from individual patients. Lancet 2000;355:1575-81

21. Vaduganathan M, Fonarow GC, Gheorghiade M. Drug therapy to reduce early readmission risk in heart failure: ready for prime time? JACC Heart Fail $2013 ; 1: 361-4$

22. Felker GM, Mentz RJ. Diuretics and ultrafiltration in acute decompensated heart failure. J Am Coll Cardiol 2012;59:2145-53

23. Bradley EH, Sipsma H, Horwitz LI, et al. Hospital strategy uptake and reductions in unplanned readmission rates for patients with heart failure: a prospective study. J Gen Intern Med 2015;30:605-11 
24. Center for Drug Evaluation and Research Drug Evaluation and Research Apprlication Number: 206143Orig1s000 Risk Assesssment and Risk Mitigation Review(s). http://www.accessdata.fda.gov/drugsatfda docs/nda/2015/2061430rig1s000Risk R.pdf. Accessed 29 August 2016. 


\section{Figure legends}

Figure 1. Hospital Readmission Reduction Program hospital readmission rates for 2011 to 2014 .

Figure 2. The cumulative distribution function of all-cause readmission rate reduction for ivabradine vs standard of care (SoC).

Figure 3. Computed Hospital Readmission Reduction Program (HRRP) penalty savings in heart failure readmission penalty payments for hospitals with different excess readmission rates.

Figure 4. Tornado diagram on reduction in Hospital Readmission Reduction Program (HRRP) penalty. 


\section{Tables}

Table 1. Model inputs for national and hospital-specific characteristics. Input Parameter Estimate

National HRRP hospital characteristics

National average total annual DRG payments for all discharges

$\$ 117,000,000,000$

National average payment per HF discharge (2011-2013) $\$ 6285$

National total number of HF discharges (2011-2013) $1,097,144$

National average HF readmission rate (2011-2013) $21.73 \%$

Hospital characteristics

Hospital total annual number of HF admissions

Hospital HF excess readmission ratio 1.056

Hospital HF readmission rate $22.95 \%$

Hospital total annual HF DRG payments $\$ 942,750$

Share of this hospital from all HRRP hospitals $0.041 \%$

Hospital total annual DRG payments for all discharges $\$ 47,988,231$

DRG, diagnosis-related group; HF, heart failure; HRRP, Hospital Readmission Reduction Program. 
Table 2. Thirty-day readmission rates for patients in the SHIFT study for all-cause readmission after an admission for HF.

Patients at Risk Rate of Readmission

of Readmission Within 30 days Posterior Probabilities of RD

Treatment $\quad n \quad$ Mean $(95 \% \mathrm{Crl}) \quad \overline{p(\mathrm{RD}<-2.0 \%)} \quad p(\mathrm{RD}<0 \%)$

Ivabradine $\quad 479 \quad 15.05 \%(12.00,18.36)$

$\begin{array}{lllll}\text { Placebo } & 623 & 17.02 \%(14.17,20.09) & 50 \% & 82 \%\end{array}$

$\mathrm{Crl}$, credible interval; HF, heart failure; RD, rate difference; SHIFT, Systolic $\underline{\text { Heart }}$

Failure Treatment with the $\underline{\underline{f}}$ Inhibitor Ivabradine $\underline{\text { Irial. }}$ 
Table 3. Reduction in readmission rates due to use of ivabradine.

\begin{tabular}{lc}
\hline Parameter & Change \\
\hline Median readmission rate change for ivabradine vs SoC & $-2.03 \%$ \\
Probability to observe the median rate change & $50.0 \%$ \\
Eventual readmission rate change & $-1.01 \%$ \\
Hospital ivabradine utilization (assumption) & $100.0 \%$ \\
Hospital effective readmission rate change for eligible patients & $-1.01 \%$ \\
Hospital HF readmission rate with ivabradine* & $21.93 \%$ \\
Hospital HF excess readmission ratio without ivabradine & 1.056 \\
Hospital HF excess readmission ratio with ivabradine & 1.0093
\end{tabular}

HF, heart failure; SoC, standard of care.

${ }^{*}$ For the example hospital with an HF readmission rate of $22.95 \%$. 
Table 4. Savings in reimbursement penalties due to the use of ivabradine.

\begin{tabular}{lcc}
\hline Results & SoC & Ivabradine \\
\hline Penalty payments for risk-adjusted excess HF readmissions & $\$ 52,794$ & $\$ 8778$ \\
Hospital-specific readmission payment adjustment factor & $99.8900 \%$ & $99.9817 \%$ \\
Reduction amount for excess readmissions (97\% floor) & $\$ 52,794$ & $\$ 8778$ \\
Net savings in penalty payments & $n / a$ & $\$ 44,016$ \\
Percentage reduction in HF readmission penalty payments & $n / a$ & $83.37 \%$ \\
\hline HF, heart failure; n/a, not available; SoC, standard of care. & &
\end{tabular}


Figures

Figure 1.

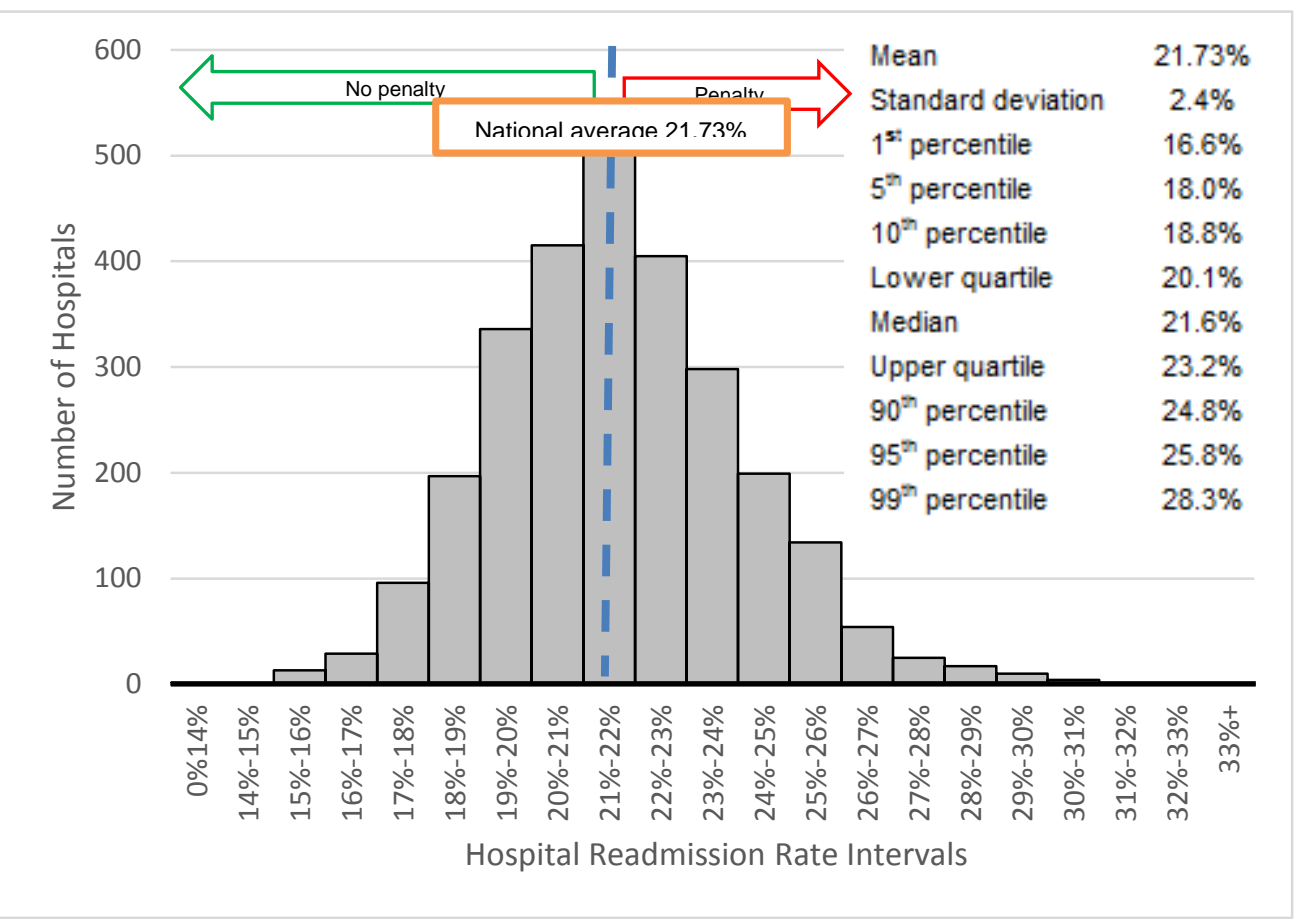


Figure 2.

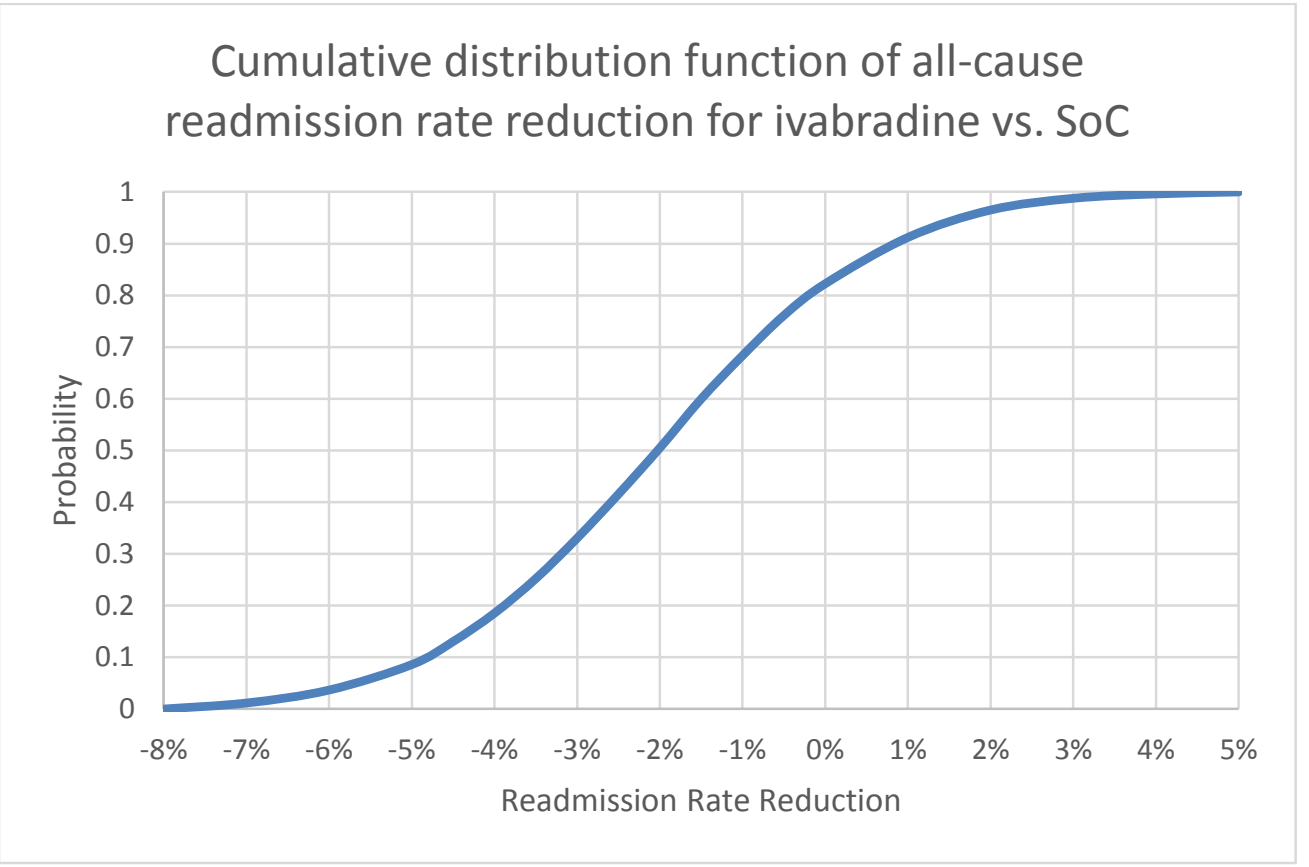


Figure 3.

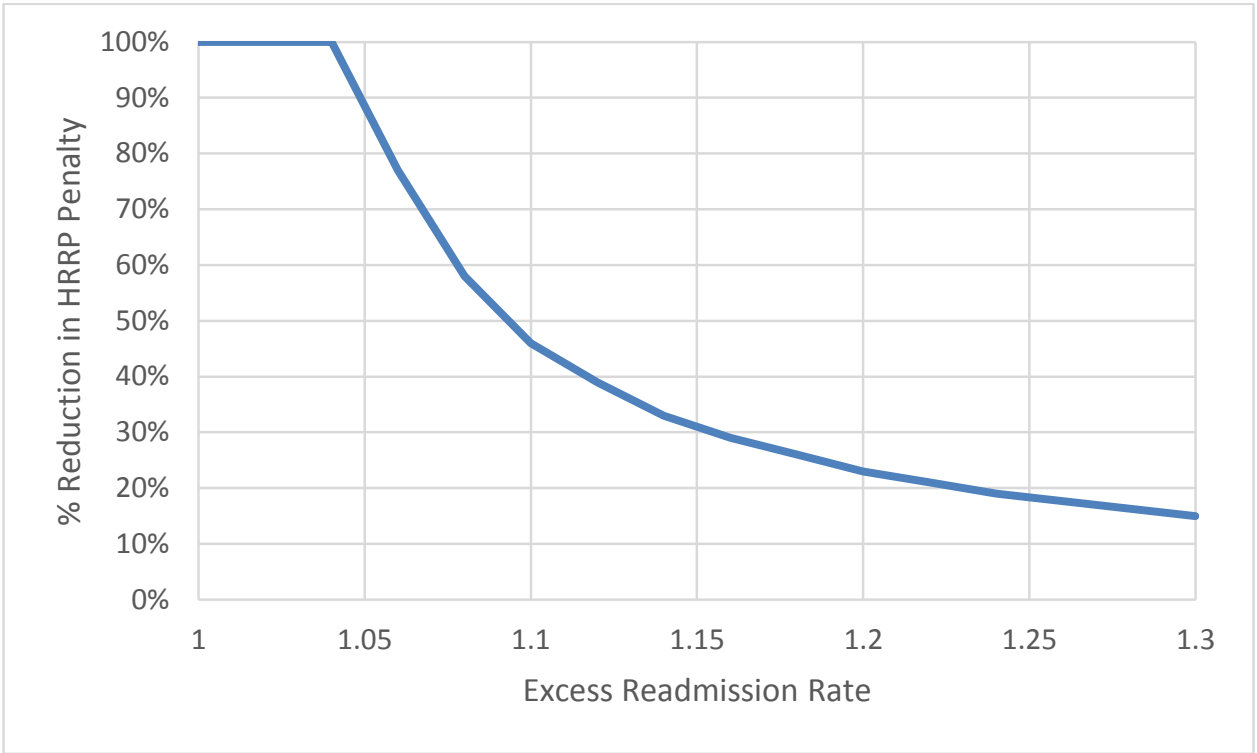


Figure 4.

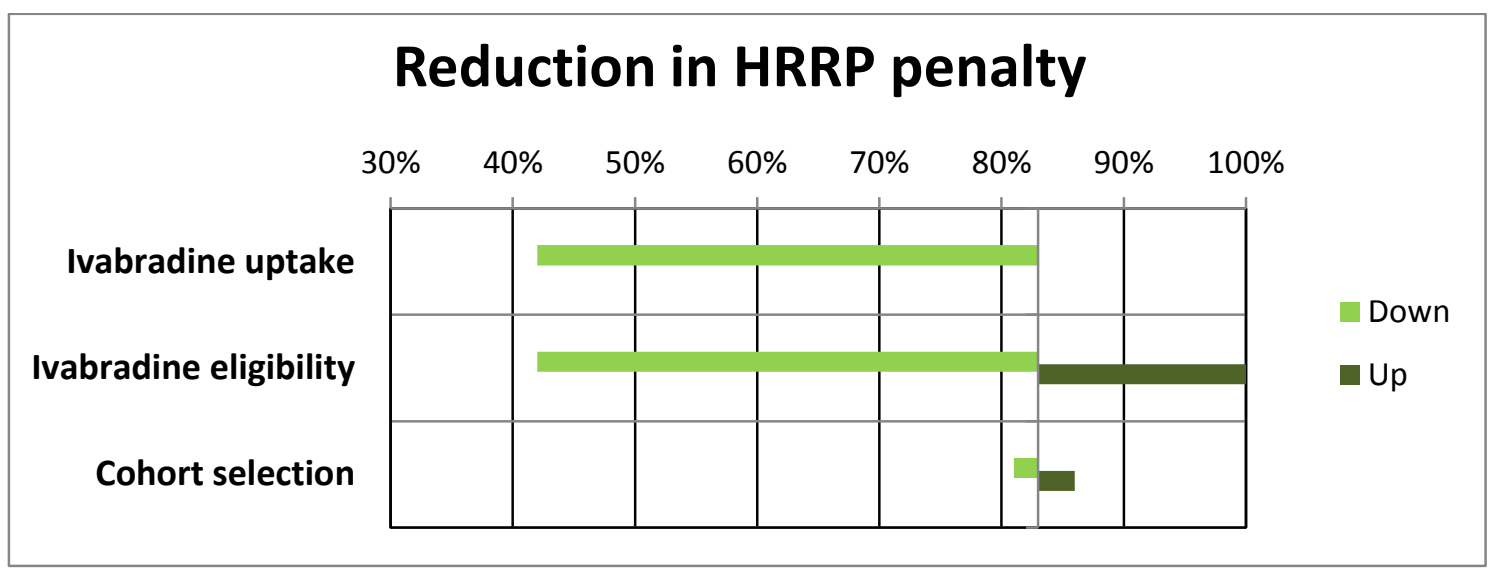

